\title{
Sea ice segmentation using Tandem-X pursuit monostatic and alternating bistatic modes
}

\author{
Temesgen G. Yitayew ${ }^{1}$, Anthony P. Doulgeris ${ }^{1}$,Torbjørn Eltoft ${ }^{1}$, Wolfgang Dierking ${ }^{2}$, Camilla Brekke ${ }^{1}$, and Anja Rösel ${ }^{3}$ \\ ${ }^{1}$ UIT, The Arctic University of Norway, ${ }^{2}$ AWI, Alfred Wegener Institute, Germany, ${ }^{3}$ NPI, Norwegian Polar Institute \\ \{temesgren.g.yitayew, anthony.p.doulgeris, torbjorn.eltoft, camilla.brekke\} @uit. no \\ Wolfgang.Dierking@awi.de, Anja.Rosel@npolar.no
}

\begin{abstract}
In this paper we investigate interferometric pairs of SAR images acquired by Tandem-X with the monostatic pursuit and the alternating bistatic modes for sea ice segmentation. The individual SAR images are modelled as non-Gaussian, and from the modelled data different features are extracted, stacked together and clustered. The interferometric coherence is regarded as an additional feature and utilized for clustering. In addition to complementing the information extracted from the individual images, the interferometric coherence is found to be capable of discriminating between open water and sea ice, as well as between different ice types.
\end{abstract}

\section{INTRODUCTION}

In recent years, several algorithms for segmentation of multichannel SAR images have been developed [1], [2], [3]. So far, the algorithms have been applied to various categories of polarimetric data, i.e. dual-, quad- or compact polarization data. The algorithms are generally based on two distinct and divergent approaches - one using highly flexible textured models for the covariance matrix data (such as the Wishart, $\mathrm{K}$-Wishart, and U-distribution), and the other using simple features extracted from such data (the Extended Polarimetric Feature Space method). However, the algorithms are generic in the sense that they can be used to analyze data of any polarimetric dimension, and multi-dimensional images generated by stacking time series of co-located images.

Synthetic Aperture Radar interferometry (InSAR) is a technique that uses the phase difference between two co-registered complex SAR images acquired from slightly shifted positions (or different times or both) to accurately estimate either surface topography or small target movements. The interferometric coherence is a parameter computed in a small moving and averaging window by taking the absolute value of the complex correlation coefficient of the SAR image pair [4]. It is primarily used to quantitatively describe the phase stability between the two acquisitions [4], [5]. The InSAR coherence has also been used to study the properties of different types of media such as land surfaces containing glacier, valleys and shore lines [6], and fast ice in the baltic sea [7], [8]. However, most of previous studies deal with temporal baselines on the scale of several hours and days. This work deals with segmentation of interferometric pair images acquired using Tandem-X in a pursuit monostatic and alternating bistatic modes over sea ice in the Fram strait area, close to Greenland. The temporal baselines from the two datasets are in the order of seconds and milliseconds respectively. As it is pointed out, the coherence is an indicator for the degree of correlation between the two acquisitions. This decorrelation is a function of the spatial and temporal baselines, as well as the Signal to Noise Ratios of the two SAR systems [5]. The sea ice cover is complex and dynamic, and may be composed of, e.g., different ice types, deformation structures, frozen leads, open water areas, and melt ponds. The actual ice conditions are influenced by the meteorological and environmental conditions. Depending on the temporal and spatial baseline scales, all these different structures in a given sea ice scene are expected to have different degree of decorrelation. This in turn is expected to give rise to different contrasting regions in the coherence image, and in this work, we aim to take advantage of this fact to investigate the potential of the coherence for segmenting sea ice. The segmentation approach is based on combining simple features extracted from the individual images, together with the coherence extracted from the InSAR pair. The main objectives are to investigate the value added by the interferometric coherence with respect to features extracted from the individual images, and to identify the optimal combination of the features considered for improved segmentation analysis.

\section{DATASETS}

The InSAR dataset considered in this study consists of two sets. The first one contains Tandem-X data acquired over the the Fram strait region using the pursuit monostatic mode on the $13^{\text {th }}$ of January, 2015. In this mode of acquisition, the two satellites (TerraSAR-X and Tandem-X) are operated independently, yet the orbits are adjusted to avoid interference between the radar signals, and to keep the orbits in close formation to make InSAR data-takes possible [9]. The two interferometric pair images are 10 seconds apart in time, acquired at $\mathrm{HH}$ polarization with an incidence angle of $32^{\circ}$. The perpendicular baseline to the line-of-sight (the projection of the across-track baseline perpendicular to the slant range) for this interferometric pair is $284.6 \mathrm{~m}$. The two co-registered and multi-looked SAR images that form the InSAR pair are shown in Fig. 1(a) and (b). As it can be seen from the figure, the scene appears to contain leads with both open water and newly formed sea ice. The interferometric coherence from this set is shown in Fig. 1(c).

The second set is a separate dataset from a different region (also in the Fram strait), acquired by Tandem-X on the $2^{\text {nd }}$ of September, 2016, but using the alternating bistatic mode. This dataset was collected during the 2016 Fram strait cruise campaign organaized by the Norwegian polar Institute. In the alternating bistatic mode of acquisition, the transmitter is switched on a pulse-to-pulse basis and the two satellites receive at the same time in a synchronized operation mode. Consequently, two monostatic and two bistatic SAR images are obtained. This leads to the formation of three interferometric pairs, where two of them are formed by combining the monostatic and bistatic channels relative to each master satellite 


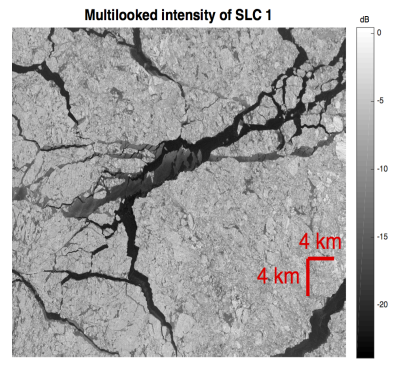

(a)

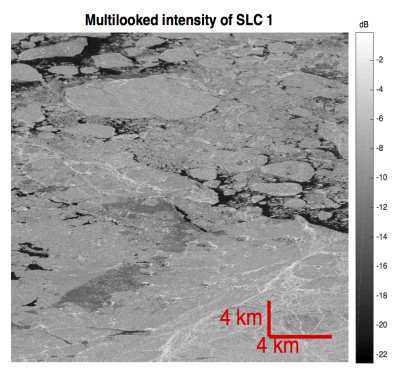

(d)

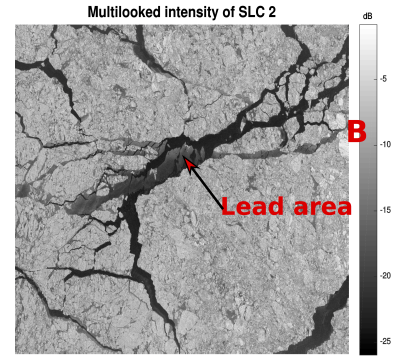

(b)

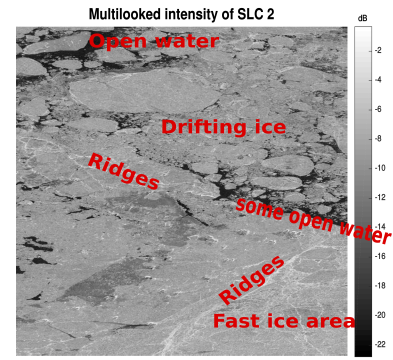

(e)

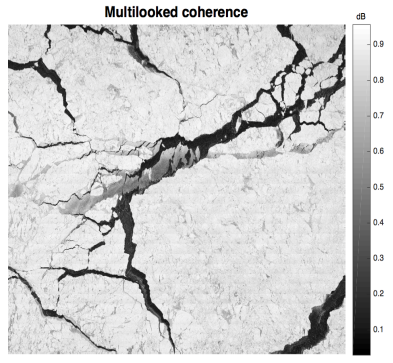

(c)

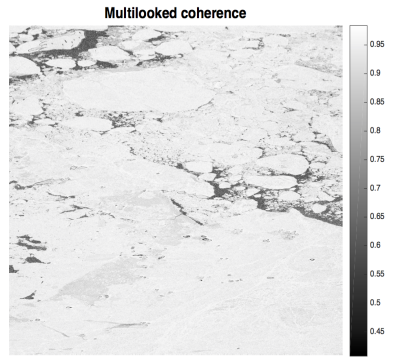

(f)

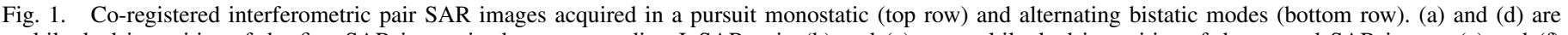

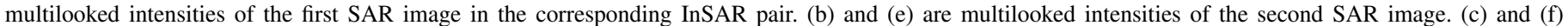
are the corresponding InSAR coherence images. SLC stands for Single Look Complex data.

acquisition, and the third one is formed by combining the two monostatic channels [9]. The InSAR pair which is obtained by combining the two monostatic channels has a better phase-toheight sensitivity than the ones obtained from the combination of the monostatic and bistatic channels. Details regarding the different modes of acquisition of Tandem-X can be found in [9]. In this study, only the InSAR pair formed from the combination of the two monostatic images is considered. The two images are acquired at $\mathrm{VV}$ polarization, with an incidence angle of $39.7^{\circ}$. The perpendicular baseline for this interferometric pair is $411.64 \mathrm{~m}$, whereas the along track baseline is $213 \mathrm{~m}$, which correspond to a temporal baseline of 0.0304 seconds. The baselines in both acquisition modes satisfy the critical baseline requirements [10]. The two coregistered and multi-looked SAR images that form this second InSAR pair are shown in Fig. 1(d) and (e). As it is confirmed from photos and observations from helicopter flights over the area, this second sea ice scene contains fast ice with ridges (in the lower right) and slowly drifting ice in the top part of the scene. The interferometric coherence from this set is shown in Fig. 1(f).

\section{RESULTS AND DISCUSSIONS}

The intensity data in a SAR image reveal a non-Gaussian distribution, and can be modelled by using the so called product model, i.e., the product of a non-Gaussian texture term and a Gaussian speckle term [1], [2], [3]. From the modelled multilooked complex (MLC) data, a number of simple features can be extracted [1], [3].

In this study, three types of features are considered. The first is Relative Kurtosis (RK) which is used as a measure of non-Gaussianity and represents the texture of the individual SAR images, the second one is the absolute backscatter which represents the radar cross section of the individual SAR images and the third one is the interferometric coherence from the pair. Details regarding the first two features can be found in [1], [3]. The interferometric coherences shown in Fig. 1 are computed after all the standard interferometric preprocessing steps such as co-registration and flat earth phase removal is applied to the data. In total, the following five features are extracted from each of the two interferometric datasets.

- Feature 1 - RK from the $1^{\text {st }}$ image

- Feature 2 - Absolute backscatter from the $1^{\text {st }}$ image

- $\quad$ Feature 3 - RK from the $2^{\text {nd }}$ image

- Feature 4 - Absolute backscatter from the $2^{\text {nd }}$ image

- Feature 5 - Interferometric coherence

Then a clustering algorithm is applied after stacking together a few combinations of the features. The automatic clustering is achieved through a modified Expectation Maximization algorithm, with an additional Goodness of-fit test allowing splitting and merging of clusters [1], [2], [3]. The resulting image segmentation depicts the statistically significant clusters within the image.

Fig. 2 shows the segmentation results from the pursuit monostatic InSAR dataset. Fig. 2(a) and (b) show the segmentation results of using the RK and absolute backscatter of the first SAR image, respectively, whereas Fig. 2(c) shows the segmentation map using the coherence. As it can be seen from the figures, the performance of RK is relatively poor. Even though it is not shown here, the segmentation results of using feature 3 and 4, namely RK and absolute backscatter of the second SAR image is comparable to the ones obtained using the corresponding features of the first 


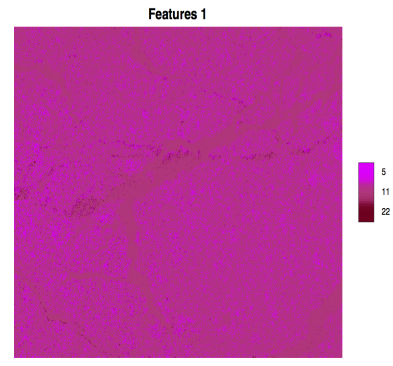

(a)



(d)

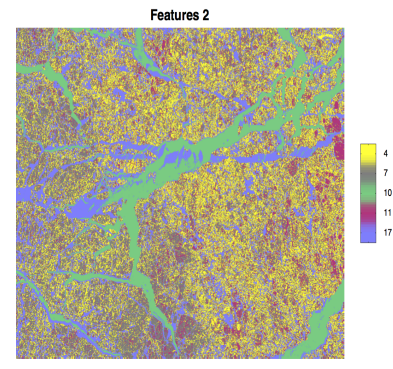

(b)

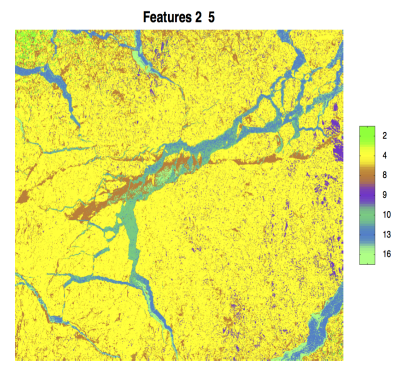

(e)

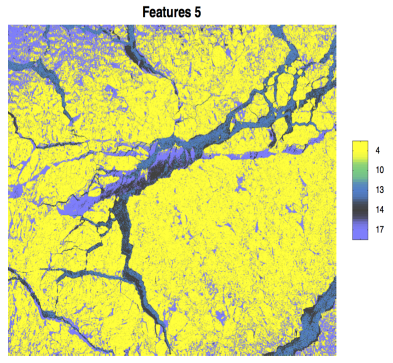

(c)



(f)

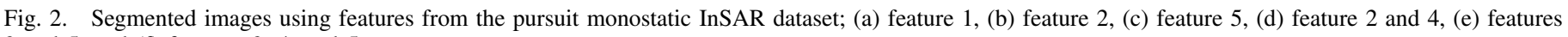
2 and 5 , and (f) features 2, 4, and 5

SAR image. Due to its poor performance, the RK of both datasets it is not further investigated. Fig. 2(d), (e) and (f) show the segmentation results of using combinations of the three features (after excluding features 1 and 3). The segmentation result based on combining the absolute backscatter of the first and second images is shown in Fig. 2(d). This result shows better discriminating capability of the combined features of the SAR image pair than the individuals. The segmentation map based on combining the coherence with absolute backscatter of the first image is shown in Fig. 2(e). From the figure, it can be clearly seen that combining the coherence with the absolute backscatter gives more distinct classes than using the coherence alone. Therefore, the interferometric coherence complements the backscatter information. As an example, the presumably open water (the very dark region in the top row of Fig. 1) and newly formed sea ice in the lead area are segmented as separate classes with the coherence [Fig. 2(c)], but not with the absolute backscatter [Fig. 2(b)]. On the other hand, the apparent brighter ice floes designated by letter "B" in Fig. 1(b) are detected and segmented as separate class with the backscatter feature [Fig. 2(b)], but not with the coherence. These observations clearly indicate the complementary nature of these two features in this particular example. The ability of the coherence to discriminate between the ice and open water can be linked to the fact that open water decorrelates faster than the ice. The decorrelation time window of water is very short and varies between 8 and 10 milliseconds at Xband [11]. The 10 second temporal baseline between these two InSAR acquisitions is much bigger than this time window, and consequently, open water is expected to be characterized by low coherence. Fig. 2(f) shows the segmentation map based on all the three features $(2,4$ and 5). As it can be seen from the figure, many of the distinct classes in the lead area identified with the coherence alone or the coherence combined with absolute backscatter also remain distinct. However, the use of all the three features resulted in too many details and it may not be feasible to link them to actual physical classes.

Fig. 3 shows the corresponding segmentation results from the alternating bistatic InSAR dataset. As it can be seen from Fig. 3(a), the performance of RK is relatively poor in this dataset too. In contrast to the previous dataset, the absolute backscatter [see Fig. 3(b)] gives clear distinct classes compared to the coherence [see Fig. 3(c)]. For example, the ridges labeled in Fig. 1(e) are clearly identified and segmented as separate class (yellow) with the absolute backscatter, whereas they look less obvious in the segmented image based on the coherence. However, the coherence still managed to discriminate open water (blue class) from the rest, showing that the coherence is a reliable feature to discriminate between open water and ice. In this dataset too, the 30 millisecond temporal baseline is bigger than the 10 millisecond decorrelation time window of water, and consequently the open water areas are characterized by low coherence values. The relatively limited performance of the coherence in the fast ice region could be linked to the fact that this region is relatively stable and characterized by relatively uniform high coherence values. Moreover, in contrast to the previous dataset, combining the backscatter features (features 2 and 4) from the two SAR images appears to add very little value compared to the segmentation performance of the individuals [compare Fig. 3(b) and 3(d)]. For this particular dataset, segmenting the backscatter intensity [see Fig. 3(b)] appears to be enough to identify the major classes.

In summary, the performance of RK in both datasets is relatively poor. In the first dataset, the coherence performs better than the backscatter features and complements the backscatter for segmentation. However, in the second dataset, it does not outperform the backscatter and it does not significantly complement the backscatter. This indicates that, this complementarity nature of the coherence for sea ice segmentation 


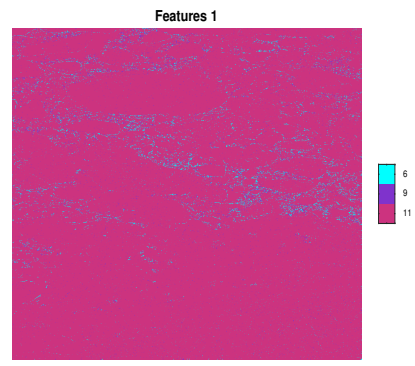

(a)

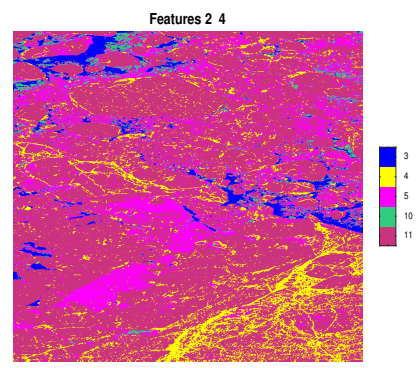

(d)

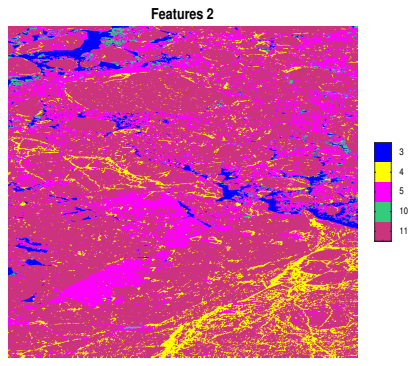

(b)

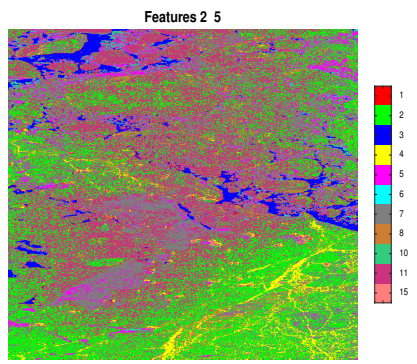

(e)

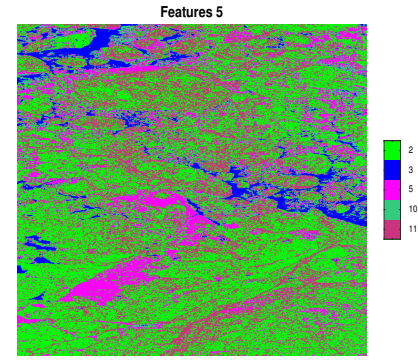

(c)

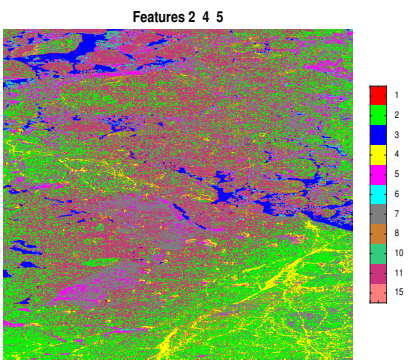

(f)

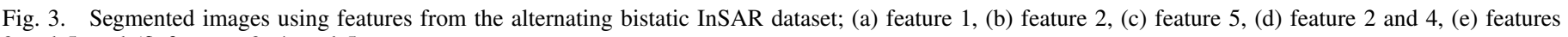
2 and 5 , and (f) features 2,4 , and 5 .

is conditional. Whenever the scene is dominated by features such as leads, newly formed ice and open water that give rise to contrasting coherence values, the coherence may play an important role for identifying and segmenting the different classes. However, whenever the scene is dominated by stable features such as fast ice and ridges which are characterized by high and relatively uniform coherence values, the coherence does not add a significant value for segmentation. In both datasets, the temporal baseline is longer than the decorrelation time window of water, and consequently the coherence has managed to discriminate open water from the rest. As it is shown in the second dataset, using many features does not necessarily add value for segmentation. Sometimes, the backscatter intensity image could be enough to identify the major classes.

\section{CONCLUSion}

In this work, an interferometric dataset acquired by Tandem- $\mathrm{X}$ in the monostatic pursuit and the alternating bistatic modes are investigated for sea ice segmentation. Three different features are considered and two of them, namely the absolute backscatter and the InSAR coherence are found to have good capability of identifying the different classes of the scenes considered. The complementarity nature of the coherence and the backscatter intensity for sea ice segmentation is investigated, and it is found that the degree of complementarity could vary from scene to scene. In both datasets, the coherence has managed to discriminate open water from the rest of the scene.

\section{ACKNOWLEDGMENT}

The authors would like to acknowledge CIRFA (RNC Grant no. 237906). Tandem-X data was collected in part under the proposal ID OTHER6835.

\section{REFERENCES}

[1] A. P. Doulgeris and T. Eltoft, "Scale mixture of gaussian modelling of polarimetric sar data," EURASIP Journal on Advances in Signal Processing, vol. 2010, no. 1, p. 1, 2009.

[2] A. P. Doulgeris, S. N. Anfinsen, and T. Eltoft, "Automated nonGaussian clustering of polarimetric synthetic aperture radar images," IEEE Transactions on Geoscience and Remote Sensing, vol. 49, no. 10, pp. 3665-3676, 2011.

[3] A. P. Doulgeris and T. Eltoft, "Polsar image segmentation-advanced statistical modelling versus simple feature extraction," in 2014 IEEE Geoscience and Remote Sensing Symposium. IEEE, 2014, pp. 10211024.

[4] P. A. Rosen, S. Hensley, I. R. Joughin, F. K. Li, S. N. Madsen, E. Rodriguez, and R. M. Goldstein, "Synthetic aperture radar interferometry," Proceedings of the IEEE, vol. 88, no. 3, pp. 333-382, 2000.

[5] H. A. Zebker and J. Villasenor, "Decorrelation in interferometric radar echoes," IEEE Transactions on geoscience and remote sensing, vol. 30, no. 5, pp. 950-959, 1992.

[6] D. J. Weydahl, "Analysis of ERS tandem SAR coherence from glaciers, valleys, and fjord ice on Svalbard," IEEE Transactions on Geoscience and Remote Sensing, vol. 39, no. 9, pp. 2029-2039, 2001.

[7] P. Dammert, M. Lepparanta, and J. Askne, "Sar interferometry over baltic sea ice," International Journal of Remote Sensing, vol. 19, no. 16, pp. 3019-3037, 1998.

[8] A. Berg, P. Dammert, and L. E. Eriksson, "X-band interferometric sar observations of baltic fast ice," IEEE Transactions on Geoscience and Remote Sensing, vol. 53, no. 3, pp. 1248-1256, 2015.

[9] G. Krieger, A. Moreira, H. Fiedler, I. Hajnsek, M. Werner, M. Younis, and M. Zink, "Tandem-x: A satellite formation for high-resolution sar interferometry," IEEE Transactions on Geoscience and Remote Sensing, vol. 45, no. 11, pp. 3317-3341, 2007.

[10] W. Dierking, O. Lang, and T. Busche, "Sea ice local surface topography from single-pass satellite insar measurements: a feasibility study," The Cryosphere, vol. doi:105194/tc-2017-40, 2017, 2017, under review.

[11] R. Romeiser and D. R. Thompson, "Numerical study on the along-track interferometric radar imaging mechanism of oceanic surface currents," IEEE transactions on geoscience and remote sensing, vol. 38, no. 1, pp. 446-458, 2000. 